\title{
Analytical approximate solutions for conservative nonlinear oscillators by modified rational harmonic balance method
}

\author{
A. Beléndez*, E. Gimeno, M.L. Álvarez, M.S. Yebra and D.I. Méndez \\ Departamento de Física, Ingeniería de Sistemas y Teoría de la Señal, Universidad de Alicante, \\ Alicante, Spain

\begin{abstract}
An analytical approximate technique for conservative nonlinear oscillators is proposed. This method is a modification of the generalized harmonic balance method in which analytical approximate solutions have a rational form. This approach gives us not only a truly periodic solution but also the frequency of motion as a function of the amplitude of oscillation. Three truly nonlinear oscillators including the cubic Duffing oscillator, fractional-power restoring force and anti-symmetric quadratic nonlinear oscillators are presented to illustrate the usefulness and effectiveness of the proposed technique. We find that this method works very well for the cubic oscillator, and excellent agreement of the approximate frequencies with the exact one has been demonstrated and discussed. For the second-order approximation, we have shown that the relative error in the analytical approximate frequency is as low as $0.0046 \%$. We also compared the Fourier series expansions of the analytical approximate solution and the exact one. This has allowed us to compare the coefficients for the different harmonic terms in these solutions. For the other two nonlinear oscillators considered, the relative errors in the analytical approximate frequencies are 0.098 and $0.066 \%$, respectively. The most significant features of this method are its simplicity and its excellent accuracy for the whole range of oscillation amplitude values, and the results reveal that this technique is very effective and convenient for solving conservative truly nonlinear oscillatory systems.
\end{abstract}

Keywords: truly nonlinear oscillators; approximate solutions; generalized harmonic balance method

AMS Subject Classification:

\section{Introduction}

Nonlinear oscillator models have been widely used in many areas not only in physics and engineering, but also they are of significant importance in other areas. Physical and mechanical oscillatory systems are often governed by nonlinear differential equations. In many cases, it is possible to replace a nonlinear differential equation by a corresponding linear differential equation that approximates the original nonlinear equation closely to give useful results [34]. Often such linearization is not feasible and for this situation the original nonlinear differential equation itself must be directly dealt with. There are a large variety of approximate methods commonly used for solving nonlinear oscillatory systems. The most common and most widely studied of all approximation methods for nonlinear differential equations are perturbation methods $[1,2,25,34,38,47]$. Some of the other techniques include variational [18,20,28,30,41,44], decomposition [42], homotopy perturbation [5,6,8,11-13,15,19,24,26,39,45], homotopy analysis [31,51], harmonic balance [34],

*Corresponding author. Email: a.belendez@ua.es

ISSN 0020-7160 print/ISSN 1029-0265 online

(C) 2008 Taylor \& Francis

DOI: $10.1080 / 00207160802380942$

http://www.informaworld.com

Techset Composition Ltd, Salisbury

GCOM338261.TeX Page\#: 15 Printed: 5/9/2008 
standard and modified Lindstedt-Poincaré [21-23,27,34,40,43], artificial parameter [27,43], parameter expanding $[29,49]$, equivalent linearization $[7,16]$, linearized and quasilinearized harmonic balance $[3,4,9,14,32,50]$ methods, etc. Surveys of the literature with numerous references and useful bibliography and a review of these methods can be found in detail in [1,2,25,27,32,34]. These nonlinear equations can be also solved using an exponential fitting method proposed by Vigo-Aguiar et al. [46-48].

The method of harmonic balance is a well-established procedure for determining analytical approximations to the solutions of differential equations, the time domain response of which can be expressed as a Fourier series. In the usual harmonic balance methods (HBM), the solution of a nonlinear system is assumed to be of the form of a truncated Fourier series [34]. This method can be applied to nonlinear oscillatory systems where the nonlinear terms are not small and no perturbation parameter is required. Being different from the other nonlinear analytical methods, such as perturbation techniques, the HBM does not depend on small parameters, such that it can find a wide application in nonlinear problems without linearization or small perturbations. Various generalizations of the HBM have been made and one of them is the rational representation proposed by Mickens and coworkers [17, 33,34,36]. In this paper, a modified generalized, rational HBM is proposed for constructing approximate analytical solutions to conservative nonlinear oscillations in which the nonlinear restoring-force $f(x)$ is an odd function of $x$ (i.e., $f(-x)=-f(-x)$ ); here $x$ represents the displacement measured from the stable equilibrium position. In this method, the approximate solution obtained approximates all of the harmonics in the exact solution [36], whereas the usual harmonic balance techniques provide an approximation to only the lowest harmonic components. For most cases, the application of the rational HBM leads to very complicated sets of algebraic equations with a very complex nonlinearity that have to be solved even for the second-order approximation. In an attempt to provide a better solution methodology, a modification in this technique is proposed for constructing the second-order analytical approximate solution to conservative nonlinear oscillators governed by differential equations with odd nonlinearity. The most interesting features of the proposed method are its simplicity and its excellent accuracy in a wide range of values of oscillation amplitude. We present three examples to illustrate the applicability and the effectiveness of the proposed approximate analytical solutions.

\section{Formulation and solution method}

Consider a single-degree-of-freedom, conservative nonlinear oscillator governed by the following dimensionless differential equation

$$
\frac{\mathrm{d}^{2} x}{\mathrm{~d} t^{2}}+f(x)=0
$$

subject to the initial conditions

$$
x(0)=A, \quad \frac{\mathrm{d} x}{\mathrm{~d} t}(0)=0
$$

where the nonlinear restoring-force function $f(x)$ is odd, i.e. $f(-x)=-f(-x)$, and satisfies $x f(x)>0$ for $x \in[-A, A], x \neq 0$. It is obvious that $x=0$ is the equilibrium position. The system oscillates between the symmetric bounds $[-A, A]$. The period and the corresponding solution are dependent on the oscillation amplitude $A$. 
A new independent variable $\tau=\omega t$ is introduced. Then Equations (1) and (2) can be rewritten as

$$
\begin{aligned}
& \omega^{2} \frac{\mathrm{d}^{2} x}{\mathrm{~d} \tau^{2}}+f(x)=0, \\
& x(0)=A, \quad \frac{\mathrm{d} x}{\mathrm{~d} \tau}(0)=0 .
\end{aligned}
$$

The new independent variable is chosen in such a way that the solution of Equation (3) is a periodic function of $\tau$ of period $2 \pi$. The corresponding frequency of the nonlinear oscillator is $\omega$ and both periodic solution $x(\tau)$ and frequency $\omega$ depend on the initial amplitude $A$.

Following the lowest order harmonic balance approximation, we set

$$
x_{1}(\tau)=A \cos \tau
$$

which satisfies the initial conditions in Equation (4). Substituting Equation (5) into Equation (3) and setting the coefficient of the resulting $\cos \tau$ to zero yield the first approximation to the frequency in terms of $A$

$$
\omega_{1}(A)=\sqrt{\frac{c_{1}}{A}}
$$

where

$$
c_{1}=\frac{4}{\pi} \int_{0}^{\pi / 2} f\left(x_{1}(\tau)\right) \cos \tau \mathrm{d} \tau
$$

is the first coefficient of the Fourier series expansion of function $f\left(x_{1}(\tau)\right)$

$$
f\left(x_{1}(\tau)\right)=\sum_{n=0}^{\infty} c_{2 n+1} \cos [(2 n+1) \tau]
$$

where only the odd multiples of are presented because the nonlinear function $f(x)$ is odd.

In order to determine an improved approximation, we use a generalized, rational form given by the following expression [34,36]:

$$
x_{2}(\tau)=\frac{A_{1} \cos \tau}{1+B_{2} \cos 2 \tau} .
$$

In this equation, $A_{1}, B_{2}$ and $\omega$ are to be determined as functions of the initial conditions expressed in Equation (4) and $\left|B_{1}\right|<1$. From Equation (4), we obtain $A_{1}=\left(1+B_{2}\right) A$, and Equation (9) can be rewritten as follows:

$$
x_{2}(\tau)=\frac{\left(1+B_{2}\right) A \cos \tau}{1+B_{2} \cos 2 \tau} .
$$

Substituting Equation (10) into Equation (3) leads to

$$
\omega^{2} h(\tau)+f\left(x_{2}(\tau)\right)=0
$$

where

$$
\begin{aligned}
h(\tau)= & \frac{\mathrm{d}^{2} x_{2}(\tau)}{\mathrm{d} \tau^{2}}=\frac{4 A B_{2}\left(1+B_{2}\right) \cos \tau \cos 2 \tau}{\left(1+B_{2} \cos 2 \tau\right)^{2}}-\frac{A\left(1+B_{2}\right) \cos \tau}{1+B_{2} \cos 2 \tau} \\
& -\frac{4 A B_{2}\left(1+B_{2}\right) \sin \tau \sin 2 \tau}{\left(1+B_{2} \cos 2 \tau\right)^{2}}+\frac{8 A B_{2}^{2}\left(1+B_{2}\right) \cos \tau \sin ^{2} 2 \tau}{\left(1+B_{2} \cos 2 \tau\right)^{3}}
\end{aligned}
$$


and

$$
f\left(x_{2}(\tau)\right)=f\left(\frac{\left(1+B_{2}\right) A \cos \tau}{1+B_{2} \cos 2 \tau}\right) .
$$

As $\left|B_{2}\right|<1$, we can do the following Taylor series expansions

$$
\begin{aligned}
h(\tau) & =\sum_{n=0}^{\infty} \alpha_{n}(\tau) B_{2}^{n}, \\
f\left(x_{2}(\tau)\right) & =\sum_{n=0}^{\infty} \beta_{n}(\tau) B_{2}^{n} .
\end{aligned}
$$

Before applying the HBM to Equation (11), we consider the following approximation in Equations (14) and (15)

$$
\begin{gathered}
h(\tau) \approx h_{2}(\tau)=\alpha_{0}(\tau)+\alpha_{1}(\tau) B_{2}+\alpha_{0}(\tau) B_{2}^{2}, \\
f\left(x_{2}(\tau)\right) \approx f_{2}\left(x_{2}(\tau)\right)=\beta_{0}(\tau)+\beta_{1}(\tau) B_{2}+\beta_{0}(\tau) B_{2}^{2},
\end{gathered}
$$

where

$$
\begin{aligned}
\alpha_{0}(\tau)= & -A \cos \tau, \\
\alpha_{1}(\tau)= & A(9 \cos 2 \tau-5) \cos \tau, \\
\alpha_{2}(\tau)= & -\frac{1}{2} A(17-34 \cos 2 \tau+25 \cos 4 \tau) \cos \tau, \\
\beta_{0}(\tau)= & f(A \cos \tau), \\
\beta_{1}(\tau)= & A \cos \tau(1-\cos 2 \tau) f_{x}(A \cos \tau), \\
\beta_{2}(\tau)= & -A \cos \tau \cos 2 \tau(1-\cos 2 \tau) f_{x}(A \cos \tau) \\
& +\frac{1}{2} A^{2} \cos ^{2} \tau(1-\cos 2 \tau)^{2} f_{x x}(A \cos \tau),
\end{aligned}
$$

where the subscript $x$ denotes the derivative of $f(x)$ with respect to $x$. Substituting Equations (16) and (17) into Equation (11) gives

$$
G_{2}\left(A, B_{2}, \omega, \tau\right)=\omega^{2} h_{2}\left(A, B_{2}, \tau\right)+f_{2}\left(A, B_{2}, \tau\right) \approx 0 .
$$

Expanding $F_{2}\left(A, B_{2}, \tau\right)$ in a trigonometric series yields

$$
\begin{aligned}
G_{2}\left(A, B_{2}, \omega, \tau\right)= & {\left[\omega^{2} H_{20}\left(A, B_{2}\right)+F_{20}\left(A, B_{2}\right)\right] \cos \tau } \\
& +\left[\omega^{2} H_{21}\left(A, B_{2}\right)+F_{21}\left(A, B_{2}\right)\right] \cos 3 \tau+\mathrm{HOH},
\end{aligned}
$$

where $\mathrm{HOH}$ stands for higher-order harmonics, and

$$
\begin{aligned}
& H_{20}\left(A, B_{2}\right)=\frac{4}{\pi} \int_{0}^{\pi / 2} h_{2}\left(A, B_{2}, \tau\right) \cos \tau \mathrm{d} \tau=-\frac{1}{2}\left(2+B_{2}\right) A, \\
& H_{21}\left(A, B_{2}\right)=\frac{4}{\pi} \int_{0}^{\pi / 2} h_{2}\left(A, B_{2}, \tau\right) \cos 3 \tau \mathrm{d} \tau=\frac{9}{4}\left(2+B_{2}\right) A B_{2}, \\
& F_{20}\left(A, B_{2}\right)=\frac{4}{\pi} \int_{0}^{\pi / 2} f_{2}\left(A, B_{2}, \tau\right) \cos \tau \mathrm{d} \tau, \\
& F_{21}\left(A, B_{2}\right)=\frac{4}{\pi} \int_{0}^{\pi / 2} f_{2}\left(A, B_{2}, \tau\right) \cos 3 \tau \mathrm{d} \tau .
\end{aligned}
$$


Substituting Equations (22)-(25) into Equation (21) and setting the coefficients of $\cos \tau$ and $\cos 3 \tau$ to zeros, respectively, yield

$$
\begin{aligned}
-\frac{1}{2}\left(2+B_{2}\right) A \omega^{2}+F_{20}\left(A, B_{2}\right) & =0 \\
\frac{9}{4}\left(2+B_{2}\right) A B_{2} \omega^{2}+F_{21}\left(A, B_{2}\right) & =0
\end{aligned}
$$

Solving Equations (26) and (27), we can obtain $B_{2}$ and the second-order approximate frequency $\omega$ as a function of $A$. It should be clear how the procedure works for constructing the secondorder analytical approximate solution. We will show in the following examples that this technique provides excellent analytical approximations to frequency and corresponding periodic solutions of conservative nonlinear oscillators.

\section{Illustrative examples}

In this section, we present three examples to illustrate the usefulness and effectiveness of the proposed technique.

Example 1 Truly nonlinear cubic Duffing oscillator. This oscillator is governed by the following differential equation with initial conditions

$$
\frac{\mathrm{d}^{2} x(t)}{\mathrm{d} t^{2}}+x^{3}(t)=0, \quad x(0)=A, \quad \frac{\mathrm{d} x}{\mathrm{~d} t}(0)=0 .
$$

For the oscillator above, one has $f(x)=x^{3}, f_{x}(x)=3 x^{2}$ and $f_{x x}(x)=6 x$. Equation (28) corresponds to a mechanical oscillator for which the restoring force is proportional to the cube of the displacement. As we can see, the linear term, $x$, is omitted in the equation and for all values of $x$, the motion is always nonlinear. One example of this nonlinear oscillator is the motion of a $\mathbf{Q 3}$ ball-bearing oscillating in a glass tube that is bent into a curve [27] is another example, as well as the motion of a mass attached to two identical stretched elastic wires for small amplitudes when the length of each wire without tension is the same as half the distance between the ends of the wires [4].

From Equations (5)-(7), we obtain the first analytical approximate formula for the frequency as

$$
\omega_{1}(A)=\frac{\sqrt{3}}{2} A \approx 0.866025 A .
$$

From Equations (19), (24) and (25), we obtain

$$
\begin{aligned}
& F_{20}\left(A, B_{2}\right)=\frac{3}{8}\left(2+2 B_{2}+B_{2}^{2}\right) A^{3}, \\
& F_{21}\left(A, B_{2}\right)=-\frac{1}{8}\left(2-3 B_{2}-6 B_{2}^{2}\right) A^{3} .
\end{aligned}
$$


Substituting Equations (30) and (31) into Equations (26) and (27) and solving for $B_{2}$ and $\omega$ yield the second analytical expression for the frequency as

$$
\begin{aligned}
B_{2} & =\frac{1}{27}\left[-14-\frac{118(4)^{1 / 3}}{(2435+27 \sqrt{12641})^{1 / 3}}+(4870+54 \sqrt{12641})^{1 / 3}\right]^{1 / 3} \approx-0.0900126 \\
\omega_{2}(A) & =A \sqrt{\frac{3\left(2+2 B_{2}+B_{2}^{2}\right)}{4\left(2+B_{2}\right)}} \approx 0.84725206 A .
\end{aligned}
$$

Therefore, the second approximation to the periodic solution of the nonlinear oscillator is given by the following equation:

$$
\frac{x_{2}(t)}{A}=\frac{0.9099874 \cos (0.84725206 A t)}{1-0.0900126 \cos (1.69450412 A t)} .
$$

This periodic solution has the following Fourier series expansion:

$$
\frac{x_{2}(t)}{A}=\sum_{n=0}^{\infty} a_{2 n+1} \cos \left[(2 n+1) \omega_{2} t\right]
$$

where

$$
a_{2 n+1}=\frac{4}{\pi} \int_{0}^{\pi / 2} \frac{0.9099874 \cos \tau}{1-0.0900126 \cos 2 \tau} \cos [(2 n+1) \tau] \mathrm{d} \tau .
$$

As we can see, Equation (34) gives an expression that approximates all of the harmonics in the exact solution, whereas the usual harmonic balancing techniques provide an approximation to only the lowest harmonic components.

We illustrate the accuracy of the modified approach by comparing the approximate solutions previously obtained with the exact frequency $\omega_{\mathrm{ex}}$ and other results in the literature. In particular, we will consider the solution of Equation (28) by means of the homotopy perturbation method (HPM) [6], the standard HBM [34] and a linearized HBM [50]. The last method incorporates salient features of both Newton's method and the HBM.

Direct integration of Equation (1) yields the exact frequency as [6]

$$
\omega_{\mathrm{ex}}(A)=\frac{\pi A}{2 K(1 / 2)}=0.847213085 A,
$$

where $K(m)$ is the complete elliptical integral of the first kind [37]. The exact solution to Equation (28) is [6]

$$
\frac{x_{\mathrm{ex}}(t)}{A}=\mathrm{cn}\left(A t ; \frac{1}{2}\right)
$$

where $\mathrm{cn}$ is the Jacobi elliptic function which has the following Fourier expansion [6,34]:

$$
\operatorname{cn}(u ; m)=\frac{2 \pi}{\sqrt{m} K(m)} \sum_{n=0}^{\infty} \frac{q^{n+1 / 2}}{1+q^{2 n+1}} \cos \left[\frac{(2 n+1) \pi u}{2 K(m)}\right],
$$

where

$$
q(m)=\exp \left[-\frac{\pi K\left(m^{\prime}\right)}{K(m)}\right]
$$


and $m^{\prime}=1-m$. With these results, the Fourier expansion of Equation (40) becomes

$$
\begin{aligned}
\frac{x_{\mathrm{ex}}(t)}{A}= & \operatorname{cn}\left(A t ; \frac{1}{2}\right)=\frac{2 \pi \sqrt{2}}{K(1 / 2)} \sum_{n=0}^{\infty}\left(\frac{\exp [(n+1 / 2) \pi]}{1+\exp [(2 n+1) \pi]}\right) \cos \left[(2 n+1) \omega_{\mathrm{ex}} t\right] \\
= & 0.95501 \cos \omega_{\mathrm{ex}} t+0.043050 \cos 3 \omega_{\mathrm{ex}} t+0.0018605 \cos 5 \omega_{\mathrm{ex}} t \\
& +0.0000804 \cos 7 \omega_{\mathrm{ex}} t+\cdot
\end{aligned}
$$

By applying the first and the second approximation based on the HBM, Mickens [34] achieved the following expressions for the frequency:

$$
\begin{aligned}
& \omega_{M 1}(A)=\frac{\sqrt{3}}{2} A=0.86602540 A, \quad \text { Relative error }=2.2 \%, \\
& \omega_{M 2}(A)=0.8507 A, \quad \text { Relative error }=0.41 \%,
\end{aligned}
$$

and for the second-order approximate solution, he obtained

$$
x_{M 2}(t)=0.9569 A \cos \omega_{M 2} t+0.0431 A \cos 3 \omega_{M 2} t .
$$

Wu et al. [50] approximately solved Equation (28) using an improved harmonic balance method (LHBM), which incorporates salient features of both Newton's method and the HBM. They achieved the following results for the first, second and third approximation orders

$$
\begin{aligned}
& \omega_{\mathrm{WSL} 1}(A)=\frac{\sqrt{3}}{2} A=0.86602540 A, \quad \text { Relative error }=2.2 \%, \\
& \omega_{\mathrm{WSL} 2}(A)=\sqrt{\frac{23}{32}} A=0.84779125 A, \quad \text { Relative error }=0.068 \%, \\
& \omega_{\mathrm{WSL} 3}(A)=\sqrt{\frac{65856986475}{91739270448}} A=0.84727284 A, \quad \text { Relative error }=0.0070 \%,
\end{aligned}
$$

and for the second-order approximate solution, they obtained

$$
\begin{aligned}
\frac{x_{\mathrm{WSL} 2}(t)}{A} & =\frac{23}{24} \cos \omega_{\mathrm{WSL} 2} t+\frac{1}{24} \cos 3 \omega_{\mathrm{WSL} 2} t \\
& =0.958333 \cos \omega_{\mathrm{WSL} 2} t+0.041667 \cos 3 \omega_{\mathrm{WSL} 2} t .
\end{aligned}
$$

Beléndez et al. [6] approximately solved Equation (28) using He's HPM. They achieved the following results for the first, second and third approximation orders:

$$
\begin{aligned}
& \omega_{B 1}(A)=\frac{\sqrt{3}}{2} A=0.86602540 A, \quad \text { Relative error }=2.2 \%, \\
& \omega_{B 2}(A)=\frac{1}{4} \sqrt{6+\sqrt{30}} A=0.84695136 A, \quad \text { Relative error }=0.031 \%, \\
& \omega_{B 3}(A)=\frac{1}{4} \sqrt{6+\sqrt{30}} A=0.84695136 A, \quad \text { Relative error }=0.031 \% .
\end{aligned}
$$

The approximate solution they obtained for the second approximation was

$$
\frac{x_{B 2}(t)}{A}=0.954538 \cos \omega_{B 2} t+0.043564 \cos 3 \omega_{B 2} t+0.0018979 \cos 5 \omega_{B 2} t .
$$


The frequency values and their relative errors obtained in this paper applying a modified generalized harmonic balance method (GHBM) are the following:

$$
\begin{aligned}
& \omega_{1}(A)=\frac{\sqrt{3}}{2} A=0.86602540 A, \quad \text { Relative error }=2.2 \%, \\
& \omega_{2}(A)=0.84725206 A, \quad \text { Relative error }=0.0046 \% .
\end{aligned}
$$

From Equations (35) and (36), the Fourier series expansion for the second-order approximate solution obtained in this paper is

$$
\begin{aligned}
\frac{x_{2}(t)}{A}= & 0.954902 \cos \omega_{2} t+0.043064 \cos 3 \omega_{2} t+0.00194209 \cos 5 \omega_{2} t \\
& +0.0000875842 \cos 7 \omega_{2} t+\cdots
\end{aligned}
$$

which has an infinite number of harmonics.

In Table 1, we present, for the second-order approximation, the comparison between the approximate and exact frequencies and the first four coefficients of the Fourier series expansions of the exact solution and the second-order the analytical approximate solution using different methods. Note that for HBM, LHBM and HPM, the number of Fourier coefficients is finite. It is clear that the second-order approximate frequency obtained in this paper is better not only than the second-order approximate frequency obtained using other approximate techniques but also than the third-order approximate frequency obtained using these methods. The normalized periodic exact solution, $x_{\mathrm{ex}} / A$, achieved using Equation (38) and the proposed second-order approximate solution, $x_{2} / A$ (Equation (34)), are plotted in Figure 1, whereas in Figure 2, we plotted the difference $\left(x_{\mathrm{ex}}-x_{2}\right) / A$. In these figures, $h$ is defined as follows:

$$
h=\frac{t}{T_{\mathrm{ex}}} .
$$

As we can see, $x_{2}(t) / A$ coincides with the exact solution $x_{\mathrm{ex}} / A$. Figures 1 and 2 show that Equations (33) and (34) can provide high accurate approximations to the exact frequency and the exact periodic solution. These results are an indication of the accuracy of the proposed modified GHBM as applied to this particular problem, and show that it provides an excellent approximation to the solution of Equation (28).

Example 2 Oscillator with fractional-power restoring force. This oscillator is governed by the following differential equation with initial conditions

$$
\frac{\mathrm{d}^{2} x(t)}{\mathrm{d} t^{2}}+x^{1 / 3}(t)=0, \quad x(0)=A, \quad \frac{\mathrm{d} x}{\mathrm{~d} t}(0)=0 .
$$

For this problem, we have $f(x)=x^{1 / 3}, f_{x}(x)=(1 / 3) x^{-2 / 3}$ and $f_{x x}(x)=-(2 / 9) x^{-5 / 3}$. This system was introduced as a model 'truly nonlinear oscillator' by Mickens [35].

Table 1. Comparison of the exact and approximate frequencies and the first four coefficients for the Fourier expansions of the exact and the second-order approximate solutions obtained using different methods.

\begin{tabular}{lllccc}
\hline & Exact solution & GHBM (this paper) & HBM [34] & LHBM [50] & HPM [6]§ \\
\hline$\omega / A$ (\% error) & 0.847213 & $0.847252(0.0046 \%)$ & $0.8507(0.41 \%)$ & $0.846779(0.068 \%)$ & $0.846951(0.031 \%)$ \\
$a_{1}(\%$ error $)$ & 0.955010 & $0.954902(0.011 \%)$ & $0.9569(0.20 \%)$ & $0.958333(0.35 \%)$ & $0.954538(0.049 \%)$ \\
$a_{3}(\%$ error $)$ & 0.043050 & $0.043064(0.033 \%)$ & $0.0431(0.12 \%)$ & $0.041667(3.2 \%)$ & $0.043564(1.2 \%)$ \\
$a_{5}(\%$ error $)$ & 0.0018605 & $0.00194209(4.4 \%)$ & 0 & 0 & $0.0018979(2.0 \%)$ \\
$a_{7}(\%$ error $)$ & 0.0000804 & $0.00008758(8.9 \%)$ & 0 & 0 & 0 \\
\hline
\end{tabular}

\footnotetext{
${ }^{\dagger} a_{2 n+1}=0(n \geq 2) .{ }^{\ddagger} a_{2 n+1}=0(n \geq 2) . \S a_{2 n+1}=0(n \geq 3)$.
} 


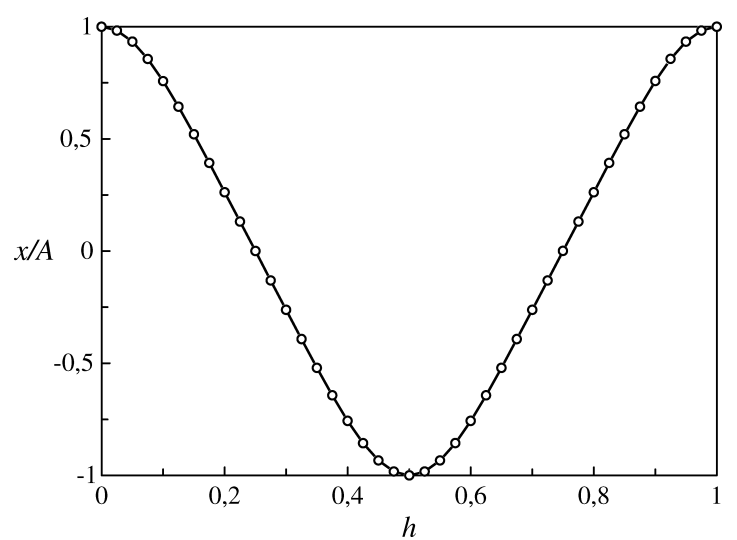

Figure 1. Comparison of normalized second-order approximate solution (circles) with the exact solution (continuous line) in Example 1.

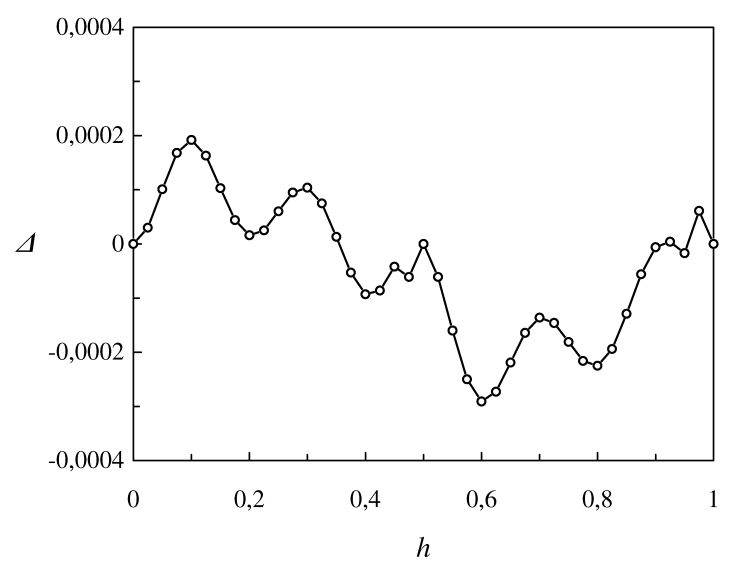

Figure 2. Difference between the normalized exact and second-order approximate solution obtained using the modified GHBM presented in this paper as a function of $h$ (Example 1).

From Equations (5)-(7), we obtain the first analytical approximate formula for the frequency as

$$
\omega_{1}(A)=\sqrt{\frac{3 \Gamma(7 / 6)}{\sqrt{\pi} \Gamma(2 / 3) A^{2 / 3}}} \approx \frac{1.07685}{A^{1 / 3}},
$$

where $\Gamma(z)$ is the Euler gamma function.

From Equations (19), (24) and (25), we obtain

$$
\begin{aligned}
& F_{20}\left(A, B_{2}\right)=\frac{3 A^{1 / 3}\left(20+4 B_{2}-B_{2}^{2}\right) \Gamma(7 / 6)}{20 \sqrt{\pi} \Gamma(2 / 3)}, \\
& F_{21}\left(A, B_{2}\right)=-\frac{3 A^{1 / 3}\left(44+55 B_{2}+2 B_{2}^{2}\right) \Gamma(7 / 6)}{220 \sqrt{\pi} \Gamma(2 / 3)} .
\end{aligned}
$$


Substituting Equations (59) and (60) into Equations (26) and (27) and solving for $B_{2}$ and $\omega$ yield the second analytical expression for the frequency as

$$
\begin{aligned}
B_{2} & \approx 0.0466088, \\
\omega_{2}(A) & =\sqrt{\frac{3\left(20+4 B_{2}-B_{2}^{2}\right) \Gamma(7 / 6)}{10 A^{2 / 3}\left(2+B_{2}\right) \Gamma(2 / 3)}} \approx \frac{1.0694051}{A^{1 / 3}} .
\end{aligned}
$$

Therefore, the second approximation to the periodic solution of the nonlinear oscillator is given by the following equation:

$$
\frac{x_{2}(t)}{A}=\frac{1.0466088 \cos \left(1.0694051 A^{-1 / 3} t\right)}{1+0.0466088 \cos \left(2.1388102 A^{-1 / 3} t\right)} .
$$

Direct integration of Equation (57) yields the exact frequency as [12]

$$
\omega_{\mathrm{ex}}(A)=\frac{2 \sqrt{\pi} \Gamma(5 / 4)}{\sqrt{6} \Gamma(3 / 4) A^{1 / 3}} \approx \frac{1.0704505}{A^{1 / 3}} .
$$

By applying the first and the second approximation based on the HBM, Mickens [35] achieved the following expressions for the frequency

$$
\begin{array}{ll}
\omega_{M 1}(A)=\frac{1.04912}{A^{1 / 3}}, & \text { Relative error }=2.0 \% \\
\omega_{M 2}(A)=\frac{1.06341}{A^{1 / 3}}, & \text { Relative error }=0.70 \%
\end{array}
$$

Wu et al. [50] approximately solved Equation (57) using an LHBM. They achieved the following results for the first and second approximation orders

$$
\begin{array}{ll}
\omega_{\mathrm{WSL} 1}(A)=\frac{1.07685}{A^{1 / 3}}, & \text { Relative error }=0.60 \%, \\
\omega_{\mathrm{WSL} 2}(A)=\frac{1.06922}{A^{1 / 3}}, & \text { Relative error }=0.12 \% .
\end{array}
$$

Beléndez et al. [12] approximately solved Equation (57) using a modified He's homotopy perturbation method (MHPM). They achieved the following results for the first and second approximation orders:

$$
\begin{array}{ll}
\omega_{B 1}(A)=\frac{1.07685}{A^{1 / 3}}, & \text { Relative error }=0.60 \% \\
\omega_{B 2}(A)=\frac{1.06861}{A^{1 / 3}}, & \text { Relative error }=0.17 \% .
\end{array}
$$

The frequency values and their relative errors obtained in this paper applying a modified GHBM are the following:

$$
\begin{array}{ll}
\omega_{1}(A)=\frac{1.07685}{A^{1 / 3}}, & \text { Relative error }=0.60 \% \\
\omega_{2}(A)=\frac{1.06941}{A^{1 / 3}}, & \text { Relative error }=0.098 \% .
\end{array}
$$

It is clear that the second-order approximate frequency obtained in this paper is better than that obtained using other approximate techniques. The comparison of the (numerical) exact normalized 


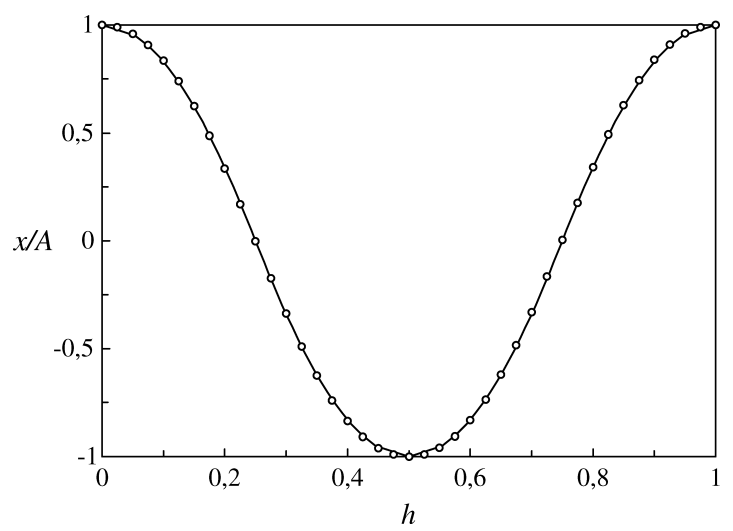

Figure 3. Comparison of normalized second-order approximate solution (circles) with the exact solution (continuous line) in Example 2.

periodic solution, $x_{\mathrm{ex}} / A$, obtained by numerically integrating Equation (57) and the proposed second-order approximate solution, $x_{2} / A$ (Equation (63)), is shown in Figure 3. This figure shows that the second approximation obtained in this paper is excellent as compared with the exact periodic solution.

Example 3 Anti-symmetric quadratic nonlinear oscillator. The anti-symmetric quadratic nonlinear oscillator is governed by the following differential equation with initial conditions

$$
\frac{\mathrm{d}^{2} x(t)}{\mathrm{d} t^{2}}+|x(t)| x(t)=0, \quad x(0)=A, \quad \frac{\mathrm{d} x}{\mathrm{~d} t}(0)=0
$$

which is an example of a nonsmooth oscillator for which $f(x)$ is a nonlinear, nonsmooth function of $x$. For this problem, we have $f(x)=|x| x, f_{x}(x)=2|x|$ and $f_{x x}(x)=2 \operatorname{sgn}(x)$, where

$$
\operatorname{sgn}(x)= \begin{cases}-1, & x<0 \\ +1, & x>0\end{cases}
$$

From Equations (5)-(7), we can easily find that the first-order approximate frequency for this oscillator is

$$
\omega_{1}(A)=\sqrt{\frac{8 A}{2 \pi}}=0.921318 \sqrt{A} .
$$

Applying Equations (19), (24) and (25) to this oscillator, we obtain

$$
\begin{aligned}
& F_{20}\left(A, B_{2}\right)=\frac{8}{105 \pi}\left(35+28 B_{2}+8 B_{2}^{2}\right) A^{2}, \\
& F_{21}\left(A, B_{2}\right)=\frac{8}{105 \pi}\left(7-20 B_{2}-24 B_{2}^{2}\right) A^{2} .
\end{aligned}
$$


Substituting Equations (76) and (77) into Equations (26) and (27) and solving for $B_{2}$ and $\omega$ yield the second analytical expression for the frequency as

$$
\begin{aligned}
B_{2}= & -\frac{17}{18}+\frac{(20155+9 \sqrt{5387191})^{1 / 3}}{4^{1 / 3} 18} \\
& -\frac{247}{18(40310+18 \sqrt{5387191})^{1 / 3}} \approx-0.0529501, \\
\omega_{2}(A)= & \sqrt{\frac{16\left(35+28 B_{2}+8 B_{2}^{2}\right) A}{105 \pi\left(2+B_{2}\right)}} \approx 0.9140759 \sqrt{A} .
\end{aligned}
$$

Therefore, the second approximation to the periodic solution of the nonlinear oscillator is given by the following equation:

$$
\frac{x_{2}(t)}{A}=\frac{0.9470499 \cos (0.9140759 \sqrt{A} t)}{1-0.0529501 \cos (1.828152 \sqrt{A} t)} \text {. }
$$

Direct integration of Equation (73) yields the exact frequency as [10]

$$
\omega_{\mathrm{ex}}(A)=\sqrt{\frac{3 \pi}{2}} \frac{\Gamma(5 / 6)}{\Gamma(1 / 3)} \sqrt{A}=0.914681 \sqrt{A} .
$$

By applying the first approximation based on the HBM and a second-order rational HBM, Mickens [10,34] achieved the following expressions for the frequency:

$$
\begin{aligned}
& \omega_{M 1}(A)=\sqrt{\frac{8 A}{2 \pi}}=0.921318 \sqrt{A}, \quad \text { Relative error }=0.73 \%, \\
& \omega_{M 2}(A)=0.914044 \sqrt{A}, \quad \text { Relative error }=0.070 \% .
\end{aligned}
$$

Beléndez et al. [10] approximately solved Equation (73) using an MHPM. They achieved the following results for the first and second approximation orders:

$$
\begin{aligned}
& \omega_{B 1}(A)=\sqrt{\frac{8 A}{2 \pi}}=0.921318 \sqrt{A}, \quad \text { Relative error }=0.73 \%, \\
& \omega_{B 2}(A)=0.914274 \sqrt{A}, \quad \text { Relative error }=0.045 \% .
\end{aligned}
$$

The frequency values and their relative errors obtained in this paper applying a modified GHBM are the following:

$$
\begin{aligned}
& \omega_{1}(A)=\sqrt{\frac{8 A}{2 \pi}}=0.921318 \sqrt{A}, \quad \text { Relative error }=0.73 \%, \\
& \omega_{2}(A)=0.914076 \sqrt{A}, \quad \text { Relative error }=0.066 \% .
\end{aligned}
$$

For this nonlinear oscillator, the second-order approximate frequency obtained using the HPM is a little better than that obtained using the rational HBM. 


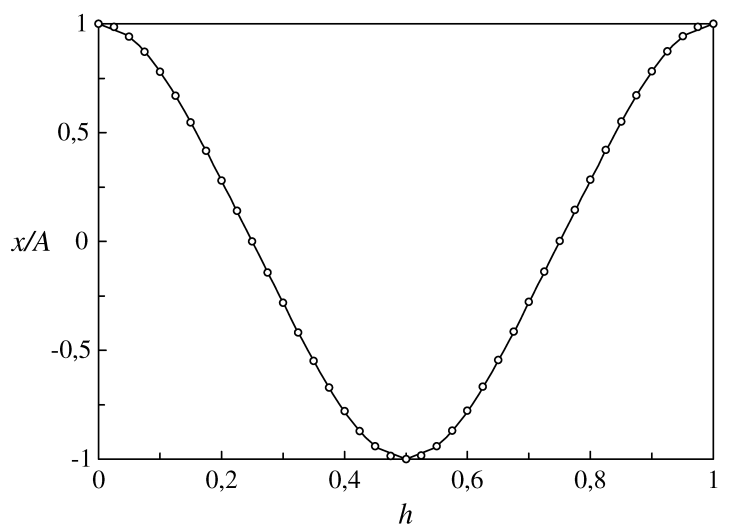

Figure 4. Comparison of normalized second-order approximate solution (circles) with the exact solution (continuous line) in Example 3.

The comparison of the (numerical) exact normalized periodic solution, $x_{\mathrm{ex}} / A$, obtained by numerically integrating Equation (73) and the proposed second-order approximate solution, $x_{2} / A$, computed by Equation (80) is shown in Figure 4. This figure show that the second approximation obtained in this paper is excellent as compared with the exact periodic solution.

\section{Conclusions}

A modified generalized, rational HBM has been applied to obtain analytical approximate solutions for nonlinear problems that are conservative and periodic. The major conclusion is that this scheme provides excellent approximations to the solution of these nonlinear systems with high accuracy. Three examples have been presented to illustrate the excellent accuracy of the analytical approximate frequencies. The analytical representations obtained using this technique give excellent approximations to the exact solutions for the whole range of values of oscillation amplitudes. For the cubic oscillator, these approximate solutions are better than that obtained using other approximate methods presented in the literature. For the second-order approximation, the relative error of the analytical approximate frequency obtained using the approach considered in this paper for the cubic oscillator is $0.0046 \%$. An interesting feature considered in this paper is the comparison between the analytical approximate solutions and the Fourier series expansion of the exact solution. This has allowed us to compare the coefficients for the different harmonics. In summary, this modified GHBM is very simple in its principle, and it can be used to solve other conservative truly nonlinear oscillators with complex nonlinearities.

\section{Acknowledgements}

This work was supported by the 'Ministerio de Educación y Ciencia', Spain, under project FIS2005-05881-C02-02. The authors express their gratitude to the reviewers for their useful suggestions and for their comments, which significantly improved the original manuscript.

\section{References}

[1] P. Amore, A. Raya, and F. M. Fernández, Alternative perturbation approaches in classical mechanics, Eur. J. Phys. 26 (2005), pp. 1057-1063. 
2] - Comparison of alternative improved perturbative methods for nonlinear oscillations, Phys. Lett. A 340 (2005), pp. 201-208.

[3] A. Beléndez and C. Pascual, Harmonic balance approach to the periodic solutions of the (an)harmonic relativistic oscillator, Phys. Lett. A 371 (2007), pp. 291-299.

[4] A. Beléndez, A. Hernández, T. Beléndez, M.L. Álvarez, S. Gallego, M. Ortuño, and C. Neipp, Application of the harmonic balance method to a nonlinear oscillator typified by a mass attached to a stretched wire, J. Sound Vib. 302 (2007), pp. 1018-1029.

[5] A. Beléndez, A. Hernández, T. Beléndez, E. Fernández, M.L. Álvarez and C. Neipp, Application of He's homotopy perturbation method to the Duffing-harmonic oscillator, Int. J. Nonlinear Sci. Numer. Simul. 8 (2007), pp. 79-88.

[6] A. Beléndez, A. Hernández, T. Beléndez, A. Márquez, and C. Neipp, Application of He's homotopy perturbation method to conservative truly nonlinear oscillators, Chaos Solitons \& Fractals 37 (2008), pp. 770-780.

[7] - An improved 'heuristic' approximation for the period of a nonlinear pendulum: linear analysis of a classical nonlinear problem, Int. J. Non-linear Sci. Numer. Simul. 8 (2007), pp. 329-334.

[8] A. Beléndez, A. Hernández, T. Beléndez, C. Neipp and A. Márquez, Application of the homotopy perturbation method to the nonlinear pendulum, Eur. J. Phys. 28 (2007), pp. 93-104.

[9] A. Beléndez, A. Hernández, A. Márquez, T. Beléndez, and C. Neipp, Analytical approximations for the period of a simple pendulum, Eur. J. Phys. 27 (2006), pp. 539-551.

[10] A. Beléndez, C. Pascual, T. Beléndez, and A. Hernández, Solution of an anti-symmetric quadratic nonlinear oscillator by a modified He's homotopy perturbation method, Nonlinear Anal. B Real World Appl. (2007), doi: j.nonrwa.2007.10.002.

[11] A. Beléndez, C. Pascual, E. Fernández, C. Neipp, and T. Beléndez, Higher-order approximate solutions to the relativistic and Duffing-harmonic oscillators by modified He's homotopy methods, Phys. Scr. 77 (2008), p. 025004.

[12] A. Beléndez, C. Pascual, S. Gallego, M. Ortuño, and C. Neipp, Application of a modified He's homotopy perturbation method to obtain higher-order approximations of a $x^{1 / 3}$ force nonlinear oscillator, Phys. Lett. A 371 (2007), pp. 421-426.

[13] A. Beléndez, C. Pascual, A. Márquez, and D. I. Méndez, Application of He's homotopy perturbation method to the relativistic (an)harmonic oscillator I: Comparison between approximate and exact frequencies, Int. J. Non-linear Sci. Numer. Simul. 8 (2007), pp. 483-491.

[14] A. Beléndez, C. Pascual, D.I. Méndez, and C. Neipp, Solution of the relativistic (an)harmonic oscillator using the harmonic balance method, J. Sound Vib. 311 (2008), pp. 1447-1456.

[15] A. Beléndez, C. Pascual, D.I. Méndez, M.L. Alvarez, and C. Neipp, Application of He's homotopy perturbation method to the relativistic (an)harmonic oscillator II: a more accurate approximate solution, Int. J. Nonlinear Sci. Numer. Simul. 8 (2007), pp. 493-504.

[16] A. Beléndez, C. Pascual, C. Neipp, T. Beléndez, and A. Hernández, An equivalent linearization method for conservative nonlinear oscillators, Int. J. Non-linear Sci. Numer. Simul. 9 (2008), pp. 9-17.

[17] K. Cooper and R.E. Mickens, Generalized harmonic balance/numerical method for determining analytical approximations to the periodic solutions of the $x^{4 / 3}$ potential, J. Sound. Vib. 250 (2002), pp. 951-954.

[18] M. Dehghan and M. Tatari, The use of He's variational iteration method for solving multipoint boundary value problems, Phys. Scripta 72 (2007), pp. 672-676.

[19] M. Gorji, D.D. Ganji, and S. Soleimani, New application of He's homotopy perturbation method, Int. J. Nonlinear Sci. Numer. Simul. 8 (2007), pp. 319-328.

[20] J.H. He, Variational iteration method - a kind of non-linear analytical technique: some examples, Int. J. Non-linear Mech. 34 (1999), pp. 699-708.

[21] - A new perturbation technique which is also valid for large parameters, J. Sound Vib. 229 (2000), pp. 1257-1263.

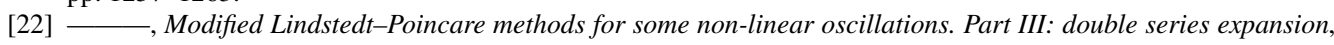
Int. J. Nonlinear Sci. Numer. Simul. 2 (2001), pp. 317-320.

[23] - Modified Lindstedt-Poincare methods for some non-linear oscillations. Part I: expansion of a constant, Int. J. Nonlinear Mech. 37 (2002), pp. 309-314.

[24] - Homotopy perturbation method for bifurcation on nonlinear problems, Int. J. Nonlinear Sci. Numer. Simul. 6 (2005), pp. 207-208.

[25] - Non-perturbative methods for strongly nonlinear problems, dissertation, de-Verlag im Internet GmbH, Berlin, 2006.

[26] - Homotopy perturbation method for solving boundary value problems, Phys. Lett. A 350 (2006), pp. 87-88.

[27] - Some asymptotic methods for strongly nonlinear equations, Int. J. Mod. Phys. B 20 (2006), pp. 1141-1199.

[28] - Variational approach for nonlinear oscillators, Chaos, Solitons \& Fractals 34 (2007), pp. 1430-1439.

[29] - Application of parameter-expanding method to strongly nonlinear oscillators, Int. J. Nonlinear Sci. Numer. Simul. 8 (2007), pp. 121-124.

[30] J.H. He and X.H. Wu, Construction of solitary solution and compact on-like solution by variational iteration method, Chaos, Solitons \& Fractals 29 (2006), pp. 108-113.

[31] S.J. Liao, An analytic aproxímate technique for free oscillations of positively damped systems with algebraically decaying amplitude, Int. J. Non-linear Mech. 38 (2003), pp. 1173-1183.

[32] C.W. Lim, B.S. Wu, and W.P. Sun, Higher accuracy analytical approximations to the Duffing-harmonic oscillator, J. Sound Vib. 296 (2006), pp. 1039-1045.

[33] R.E. Mickens, A generalization method of harmonic-balance, J. Sound Vib. 111 (1986), pp. 515-518.

[34] - Oscillations in Planar Dynamics Systems, World Scientific, Singapore, 1996. 
[35] — Analysis of non-linear oscillators having non-polynomial elastic terms, J. Sound Vib. 255 (2002), pp. 789-792.

[36] R.E. Mickens and D. Semwogerere, Fourier analysis of a rational harmonic balance approximation for periodic solutions, J. Sound Vib. 195 (1996), pp. 528-530.

[37] L. M. Milne-Thomson, Elliptic integrals, in Handbook of Mathematical Functions, M. Abramowitz and I.A. Stegun, Eds., Dover Publications, Inc., New York, 1972.

[38] A.H. Nayfeh, Problems in Perturbations, Wiley, New York, 1985.

[39] T. Özis and A. Yildirim, A comparative Study of He's homotopy perturbation method for determining frequencyamplitude relation of a nonlinear oscillator with discontinuities, Int. J. Nonlinear Sci. Numer. Simul. 8 (2007), pp. 243-248.

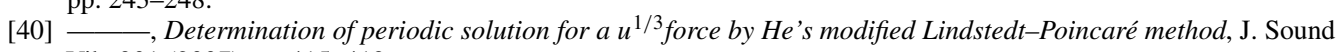
Vib. 301 (2007) pp. 415-419.

[41] M. Rafei, D.D. Ganji, H. Daniali, and H. Pashaei, The variational iteration method for nonlinear oscillators with discontinuities, J. Sound. Vib. 305 (2007), pp. 614-620.

[42] J.I. Ramos, An artificial parameter-decomposition method for nonlinear oscillators: applications to oscillators with odd nonlinearities, J. Sound Vib. 307 (2007), pp. 312-329.

[43] $\underset{1}{\longrightarrow}$ On Lindstedt-Poincaré techniques for the quintic Duffing equation, Appl. Math. Comput. (2007), in press, Q5 doi: 10.1016/j.amc.2007.03.050.

[44] - On the variational iteration method and other iterative techniques for nonlinear differential equations, Appl. Math. Comput. 199 (2008), pp. 39-69.

[45] F. Shakeri and M. Dehghan, Inverse problem of diffusion by He's homotopy perturbation method, Phys. Scr. 75 (2007), pp. 551-556.

[46] J. Vigo-Aguiar and T.E. Simos, Review of multistep methods for the numerical solution of the radial Schrödinger equation, Int. J. Quantum Chem. 103 (2005), pp. 278-290.

[47] J. Vigo-Aguiar, T.E. Simos, and J.M. Ferrándiz, Controlling the error growth in long-term numerical integration of perturbed oscillations in one or several frequencies, Proc. R. Soc. Lond. A 460 (2004), pp. 561-567.

[48] J. Vigo-Aguiar, T.E. Simos, and A. Tocino, An adapted sympletic integrator for Hamiltonian problems, Int. J. Modern Phys. C 12 (2001), pp. 225-234.

[49] S.Q. Wang and J.H. He, Nonlinear oscillator with discontinuity by parameter-expansion method, Chaos, Solitons \& Fractals 35 (2008), pp. 688-691.

[50] B.S. Wu, W.P. Sun, and C.W. Lim, An analytical approximate technique for a class of strongly non-linear oscillators, Int. J. Non-linear Mech. 41 (2006), pp. 766-774.

[51] H. Xu and J. Cang, Analysis of a time fractional wave-like equation with the homotopy analysis method, Phys. Lett. A 372 (2008), pp. 1250-1255. 